\title{
Stabilizing Morbidity and Predicting the Aesthetic Results of Radial Forearm Free Flap Donor Sites
}

\author{
Tae Kyoung Yun, Eul Sik Yoon, Duck Sun Ahn, Seung Ha Park, Byung Il Lee, Hyon Surk Kim, \\ Hi Jin You \\ Department of Plastic and Reconstructive Surgery, Korea University College of Medicine, Seoul, Korea
}

\begin{abstract}
Background The radial forearm flap is a versatile, widely used flap. However, the possibility of donor site complications has led to concern over its use. Some surgeons prefer using other flaps whose donor sites can be closed primarily with less morbidity, including avoiding unpleasant scarring. However, in our experience, donor site stability of the radial forearm flap can be reliably achieved by using well-implemented specific procedures. Here, we present a collection of donor site cases of the radial forearm flap and investigate factors that affect the aesthetic results as the basis for a reference for selecting a radial forearm flap.

Methods In this retrospective study, we reviewed 171 cases in which a radial forearm flap was used for free tissue transfer after resecting head and neck cancer. We focused on donor site morbidity rates. Each operation involved a detailed procedure designed to minimize donor site morbidity. Moreover, statistical investigations were conducted for 22 cases to determine factors affecting the scar appearance.

Results Only one case developed total skin graft necrosis as a major complication. Scar-related aesthetic results were acceptable, and the body-mass index, body weight, diabetes, and cardiac problems were significant factors related to the appearance of scars.

Conclusions Performing the radial forearm flap using a well-implemented detailed technique helps achieve acceptable donor site morbidity results. The aesthetic results were more promising for patients without excess body weight, diabetes, or cardiac problems. Therefore, anxiety about donor site morbidity should not be a reason to avoid selecting the radial forearm flap in suitable patients.
\end{abstract}

Keywords Free tissue flaps / Skin transplantation / Morbidity / Cicatrix / Aesthetics

\author{
Correspondence: Eul Sik Yoon \\ Department of Plastic and \\ Reconstructive Surgery, Korea \\ University College of Medicine, \\ 73 Inchon-ro, Seongbuk-gu, Seoul \\ 02841 , Korea \\ Tel: $+82-2-920-5368$ \\ Fax: +82-2-922-7437 \\ E-mail: yesanam2@korea.ac.kr
}

No potential conflict of interest relevant to this article was reported.

\section{INTRODUCTION}

The radial forearm free flap is a versatile flap that includes the volar forearm skin and the underlying soft tissues and fascia containing the radial artery as the perforator. It was first introduced by Yang Guofan in China in 1978. Since then, it has been commonly used as a donor flap for reconstructing the intraoral lining and resurfacing facial and neck defects [1]. It has been used in various sites, particularly for tissue defects remaining after a wide excision of head and neck malignancies. The radial forearm free flap has many advantages, including thin and pliable characteristics, a relatively hairless nature, and a long pedicle with a large external diameter, making it very useful [2]. However, the possibility of donor-site complications had led to 
concern over its use. The radial forearm free flap has conspicuous aesthetic donor site deformities induced by its large dimensions, hyperpigmentation, and scarring, which tend to concern women in particular. In addition, the skin graft placed on the donor flap site may fail and lead to an exposed tendon. Finally, some patients complain of an immobile wrist and limited hand mobility as well as other morbidities, such as sensation problems [3-5].

Surgeons who are concerned about these problems prefer to use the anterolateral thigh or latissimus dorsi flap because the donor site can be closed primarily with decreased donor site morbidity rates [6-8]. Moreover, some studies have shown better donor site morbidity rates for the ulnar forearm free flap than for the radial forearm flap [9]. However, donor site stability of the radial forearm free flap can be reliably achieved in our experience with well-implemented detailed procedures [10].

Moreover, factors that significantly affect scar deformities can be used as predictive factors when deciding among flap options for patients and surgeons strongly concerned about the shortcomings of the radial forearm flap. Several studies on postsurgical wound healing and graft failure have reported that obesity, diabetes, and peripheral vascular disease compromise wound healing $[11,12]$. However, no studies have investigated both these variables and also any other potential factors related to wound healing, graft failure, and aesthetic outcomes using statistical analysis. We speculate that some morbidities known to be associated with delayed wound healing may also relate to the appearance of scars.

In this study, we present the clinical outcomes of 171 donor site cases after head and neck reconstruction using radial forearm free flap coverage over the past 27 years and discuss procedures used for achieving minimal morbidity rates. Moreover, we evaluated aesthetic outcomes and analyzed factors associated with scarring to highlight conditions favorable for excellent results and to determine whether the radial forearm free flap is a reliable donor flap for minimizing donor site morbidity.

\section{METHODS}

We retrospectively reviewed 171 cases of radial forearm free flap coverage for reconstruction after wide excision of head and neck cancer. The operations were performed between October 1986 and February 2014. Patient data were gathered from hospital records, photographs, and other sources [10]. We focused on donor site morbidity rates.

At the same time, cases for which photographs existed and could be clearly evaluated for the scar appearance were also investigated for the overall aesthetic results. Twenty-two cases were evaluated for aesthetic results, and to identify the factors that could affect the aesthetic appearance of donor scars. Two physicians and the patients participated in the assessment of the aesthetic results. The multi-dimensional visual analog scale (VAS) was used for assessing aesthetics, and the surgeons evaluated four factors on the digital photographs (pigmentation, vascularity, acceptability, and observer comfort) as well as contours. Each patient participating in the aesthetic assessment was asked to evaluate his/her postoperative appearance. The scores were interpreted as follows: 1-2 (very poor), 3-4 (poor), 5-6 (fair), 7-8 (good), and 9-10 (excellent) [13-15]. Several variables were analyzed to identify the factors influencing the aesthetic results. The variables included in the statistical analysis are listed in Table 1. Morbidities not specifically listed were considered "others."

\section{Statistical analysis}

The statistical analysis was performed using IBM SPSS ver. 22.0 (IBM Corp., Armonk, NY, USA). The paired sample t-test was used for comparing the VAS scores of photographs rated by the physicians and the patients to determine the inter-rater reliability. The relationships between the VAS scores for aesthetic results and the other variables were examined using Pearson's product correlation analysis. Comparisons between the sexes were evaluated with the Mann-Whitney U test. Multiple linear regressions with a stepwise selection analysis were applied to confirm the significant factors from Pearson's product correlation analysis as significant factors affecting the aesthetic results. A value of $\mathrm{P}<0.05$ was considered statistically significant.

\section{Operative technique}

We primarily used left arms as donors unless they had been used previously. Preoperative preparation and intraoperative flap elevation were performed as usual.

After the flap was harvested, a split-thickness skin graft was used for closing the donor defect. The wound bed was prepared to achieve a successful skin graft and prevent donor site complications. The flexor carpi radialis and brachioradialis positions were approximated using a Vicryl 4-0 suture. We also advanced

Table 1. Variables analyzed for the correlation with the visual analog scale score for scarring

\begin{tabular}{|lc|}
\hline Type & \multicolumn{1}{c|}{ Total } \\
\hline Categorical & $\begin{array}{c}\text { Sex, diabetes, current smoking, cardiac diseases, } \\
\text { past smoking, and others }\end{array}$ \\
Numerical & Age, graft size, and smoking period \\
\hline a'Diseases not mentioned are categorized as others.
\end{tabular}


the adjacent skin flap toward the defect and quilted the wound bed margin to limit the space and minimize the wound size. If the lateral antebrachial cutaneous nerve was exposed, the radial side of the skin flap was advanced over it (Fig. 1). If the paratenon was inadequate and tendons were exposed, we advanced the flexor carpi ulnaris over it to provide a better wound bed.

The donor skin was harvested at a thickness of 14/1,000 inch, according to the forearm defect size. The graft was secured primarily with 3-0 silk sutures to prepare for a tie-over dressing. Prolene 5-0 sutures were placed in the gaps left between the silk sutures, and a quilting suture was made at the wound bed, particularly at the groove formed between the bed and the skin flap or other irregular surfaces, to prevent the formation of a hema-

\section{Fig. 1. Preparing the wound bed intraoperatively}

The locations of the flexor carpi radialis and brachioradialis were approximated. The adjacent skin flap was advanced toward the defect, and the margin was quilted at the wound bed to fully define the space and encourage a minimal wound size. If the lateral antebrachial cutaneous nerve was exposed, the radial side of the skin flap was advanced over the lateral antebrachial cutaneous nerve for protection. Vicryl 4-0 sutures were used.

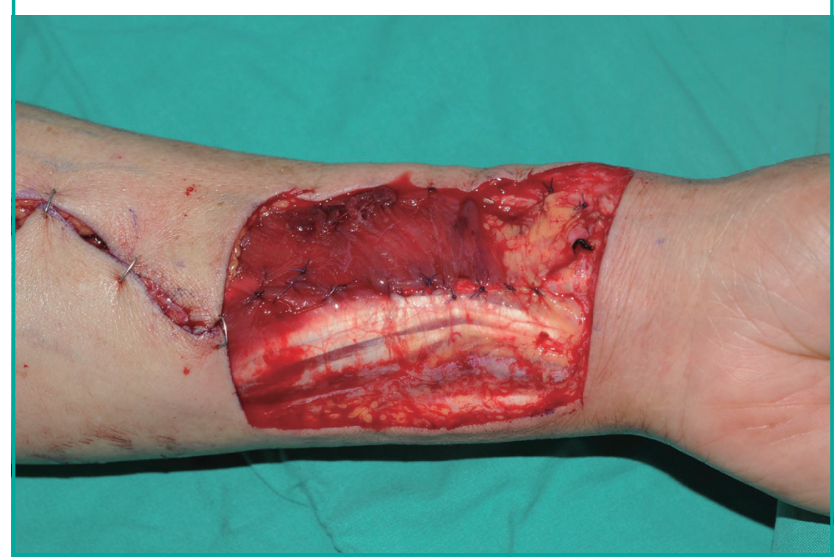

toma or seroma. The tie-over dressing was created using gauze as a traditional bolster material, or polyurethane foam (3M Reston Self-Adhering Foam Products, St. Paul, MN, USA) to compress the skin graft. Recently, we have mainly been using Reston. Notably, we tailored the compressing Reston with two layers, but not to the same size as the defect. The two layers of Reston were cut much larger than the wound area (Fig. 2A) and then squeezed to fit within the wound border. The tie-over dressing was added to maintain the squeezed state (Fig. 2B). A mild compression dressing, consisting of an elastic bandage, was applied. Finally, a splint was applied, and the patient was instructed to elevate the arm after surgery.

\section{RESULTS}

\section{Complications of donor site}

A total of 171 cases were reviewed in which a radial forearm free flap was used for cases of head and neck cancer. Of the 164 patients included, 133 were men and 31 were women. The patients were in the age group of 15-82 years (mean, 58.9 years), and most were in their 60s (Table 2). The mean follow-up period was 7.6 months. The defect donor size ranged from $3 \mathrm{~cm} \times 4$ $\mathrm{cm}$ to $8 \mathrm{~cm} \times 15 \mathrm{~cm}$ (mean, $60.6 \mathrm{~cm}^{2}$ ).

Only one major complication of total necrosis and total functional loss occurred; the patient had a seizure after the operation that resulted in a loss of the skin graft. Forty-five (26\%) cases of minor donor morbidity, such as partial necrosis, occurred. Partial necrosis of the skin graft was seen in 27 cases, all of which were successfully treated. Two patients underwent additional skin grafts, whereas the others experienced full secondary healing. A tingling sensation, numbness, decreased grip power, edema, granuloma, or hematoma was seen in some cases (Table 3).

Fig. 2. Intraoperative tie-over dressing

Polyurethane foam (Reston) was used as a bolster material for compression. (A) The Reston layers were cut much larger than the wound area. (B) The Reston layers were squeezed to fit within the wound border, and the tie-over dressing was positioned to maintain the squeezed state.
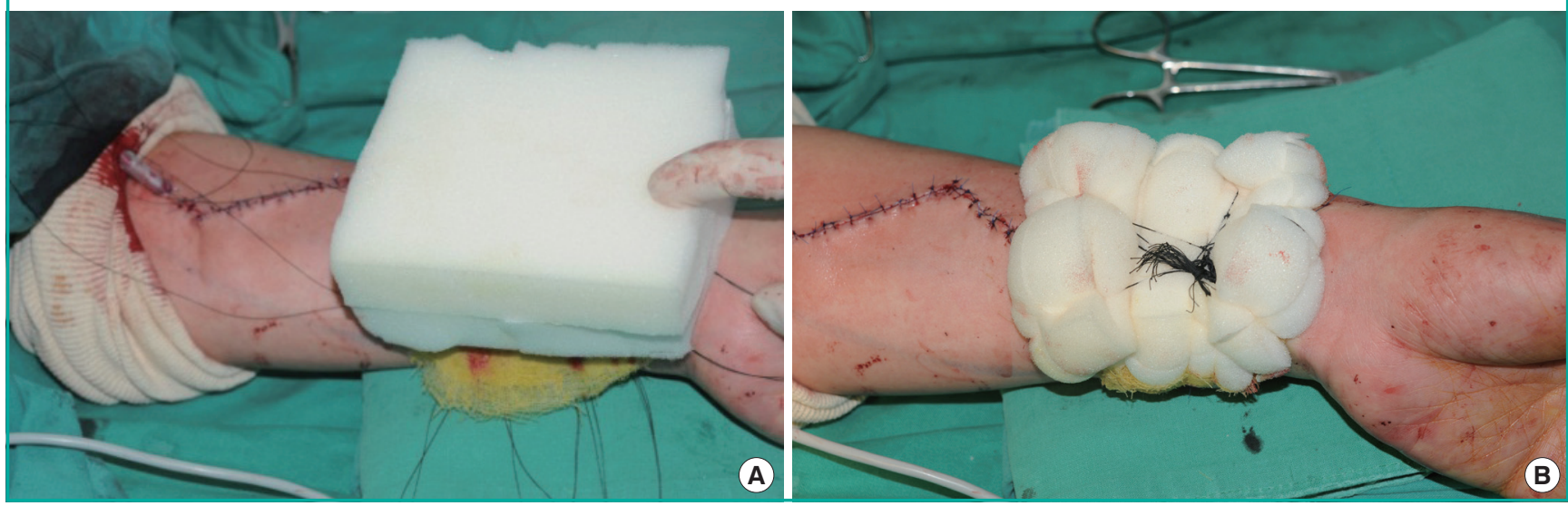
Table 2. Age and sex distribution

\begin{tabular}{|lccc|}
\hline Age $(\mathrm{yr})$ & Male & Female & Total \\
\hline $10-19$ & 1 & 0 & 1 \\
$20-29$ & 8 & 0 & 8 \\
$30-39$ & 5 & 2 & 7 \\
$40-49$ & 13 & 5 & 18 \\
$50-59$ & 54 & 12 & 66 \\
$60-69$ & 34 & 8 & 42 \\
$70-79$ & 16 & 1 & 19 \\
$80-89$ & 2 & 31 & 3 \\
Total & 133 & & 164 \\
\hline Values represent the number of patients. & & \\
\hline
\end{tabular}

Table 3. Donor site complications

\begin{tabular}{lc|}
\hline Complication & No. of cases $(\%)$ \\
\hline Total skin loss & $1(0.5)$ \\
Partial skin loss & $27(16)$ \\
Partial infection & $7(4)$ \\
Tingling sensation & $5(3)$ \\
Decreased grip power & $3(2)$ \\
Edema & $2(1)$ \\
Numbness & $1(0.5)$ \\
Granuloma & $1(0.5)$ \\
Hematoma & $1(0.5)$ \\
Total & $45(26)$ \\
\hline
\end{tabular}

One major complication (total skin loss) occurred. Partial skin loss was seen in 27 patients: two needed an additional skin graft, and the others were fully healed with conservative management. One patient had two complications and another had three complications.

\section{Aesthetic evaluation and identification of the related factors}

Twenty-two patients were enrolled for this evaluation. Aesthetic results were assessed as fair, good, or excellent in all patients (mean score, 8.05). There was no statistically significant difference between the physicians' scoring; the scores of all the cases did not differ by more than 1 point between the two raters. Further, the physician- and patient-assessed scores did not differ significantly (8.1 vs. 8.0 ), proving a satisfactory inter-rater reliability. Among all factors analyzed, the body mass index (BMI), body weight, diabetes, and cardiac diseases were negatively correlated with the aesthetic score $(\mathrm{P}<0.05)$ (Table 4$)$. The individually significant factors were also analyzed together in a stepwise multiple linear regression, and all factors were statistically significant $\left(R_{2}\right.$ value of the model $\left.=0.594 ; \mathrm{P}<0.005\right)$.

\section{DISCUSSION}

Since its introduction by Yang et al. [1] in 1978, the radial forearm flap has been used worldwide to reconstruct the defects that
Table 4. Correlation between the visual analog scale score for scarring and demographic variables

\begin{tabular}{|lcc|}
\hline Variable & $\begin{array}{c}\text { Pearson's correlation } \\
\text { coefficient }\end{array}$ & P-value ${ }^{\text {b) }}$ \\
\hline Body weight & -0.568 & $0.014^{\mathrm{b})}$ \\
Body mass index & -0.487 & $0.041^{\mathrm{b}}$ \\
Diabetes & -0.577 & $0.012^{\mathrm{b})}$ \\
Cardiac diseases & -0.513 & $0.030^{\mathrm{b})}$ \\
Current smoking & -0.096 & 0.705 \\
Past smoking & -0.037 & 0.883 \\
Smoking period & -0.125 & 0.646 \\
Age & 0.228 & 0.379 \\
Graft size & 0.024 & 0.928 \\
Othersa) & 0.000 & 1.000 \\
\hline a'Diseases not mentioned are categorized as others; ${ }^{\text {b) }} \mathrm{A}$ value of $\mathrm{P}<0.05$ was \\
considered statistically significant. & \\
\hline
\end{tabular}

remain after the resection of the head and neck cancer. However, because of donor site complications, such as conspicuous donor deformities, which are caused by large areas of hyperpigmented scars and the possibility of graft loss, some surgeons now prefer other flap choices. We are also concerned about donor site problems related to radial forearm flaps; however, we prefer to use this flap as a donor and follow meticulous procedures. As shown here, the degree of donor site morbidity was acceptable. Procedures including preserving the paratenon by meticulous dissection or approximating the flexor carpi radialis and brachioradialis locations after elevating the flap were performed to minimize morbidity, as they provide a more adherent bed for the graft than the exposed tendons [5]. Advancing the adjacent skin flap toward the defect, quilting the margin at the wound bed to facilitate coverage of the undermined space, and advancing the radial side skin flap to cover the lateral antebrachial cutaneous nerve are also useful techniques [16]. If the paratenon is inadequate for preventing tendon exposure, then the flexor carpi ulnaris can be advanced to ensure full coverage.

Recently, we also used gas-sterilized polyurethane foam (Reston) as a bolster material to provide compression with a tie-over dressing instead of other traditional methods. Wells and Kirn [17] and Saltz and Bowles [18] introduced the Reston technique as an alternative method to stabilize skin grafts and prevent hematomas and seromas, offer resistance to shear forces, provide splinting properties, and protect the wound bed from the outside environment. The main difference between our method and those of Wells and Saltz is that we used a tie-over dressing instead of fixation with a stapler. Both Wells and Saltz used foam layers of the same size as the wound, whereas we used much larger-sized sponges. In our experience, the use of a tie-over dressing increases compression and stabilizes the site. The Reston layers should be cut much larger than the wound 


\section{Fig. 3. Postoperative photographs of four other patients}

(A) Nine months after surgery. Two physicians assigned a score of 10 and 9, respectively, and the patient assigned a score of 10 . (B) Case at 11 months after surgery. Two physicians assigned a score of 9 and 8, respectively, and the patient assigned a score of 9. (C) Case at 1 year after surgery. Both the physicians and the patients assigned a score of 10. (D) Case at 8 months after surgery. Two physicians assigned a score of 9 and 8 , respectively, and the patient assigned a score of 9 .
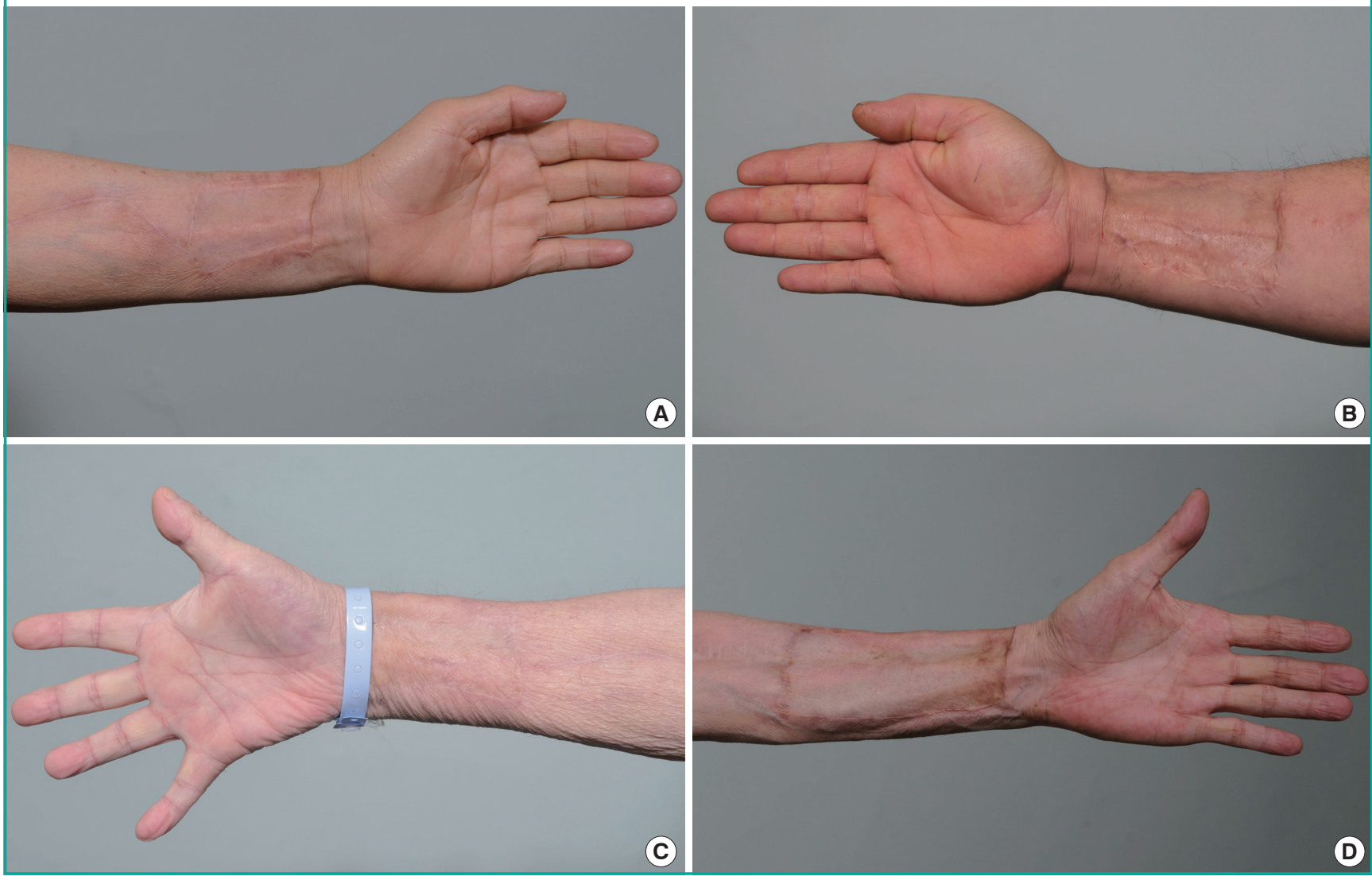

bed area when using tie-over sutures; if the Reston is the same size as the wound, the foam will be dimpled as the tie-over silk sutures are pulled, and compression will not be sufficient at the edge of the skin graft site. We also used two Reston layers to ensure adequate compression. A comparative study of using Reston as detailed was not in our scope, although in our experience, as Reston was used recently, relatively few complications have occurred.

These procedures enabled us to achieve acceptable donor site complication rates by using the radial forearm free flap. With the exception of one unusual case in which the patient had a postoperative seizure that led to total skin graft loss, we had no major complications in our 171 cases.

Our complication rates and cosmetic outcomes were relatively acceptable (Fig. 3A, D). The aesthetic outcomes evaluated by the physicians and the patients were graded as fair, good, or excellent. Lutz et al. [19] reported $98 \%$ good or fair results, whereas Toschka et al. [20] reported $94.3 \%$ good or fair results. The proportion of good or excellent results was much higher in our study. Four other studies reported a higher ratio of patients who were either not entirely satisfied or wholly dissatisfied with their aesthetic outcome, with rates of $50.6 \%, 28.4 \%, 24 \%$, and $16.7 \%$, respectively $[3,21,22]$.

Although our procedures are well-established and the results were superior to other reports, some variations in outcomes were observed. We speculate that some factors other than luck and a patient's tendency to scar may have influenced the overall outcome. Skin graft take depends on the quality of the prepared wound bed and the grafted skin, skin color match, and immobilization with dressings. Excluding the grafted skin quality, these factors have already been discussed.

The literature shows that a full-thickness graft loses approximately $40 \%$ of its original area, and a thick split-thickness graft contracts by approximately $10 \%$ as a result of primary contraction because of immediate elastic recoil $[23,24]$. If the harvested skin contracts too much, it would have to be stretched too much to cover the defect. We hypothesize that an over-stretched skin graft under excess tension would cause wound healing or scar- 
ring problems, which would result in poor aesthetic results. In our experience, at least in older populations, thinner individuals tend to have more loose skin, whereas obese people's skin is usually tighter. Thus, we speculate that skin harvested from obese individuals may shrink more. Therefore, we selected BMI and body weight as variables to investigate the relationship with aesthetic results.

Some studies have evaluated postsurgical wound healing and graft failure. Wilson and Clark [11] overviewed the impact of obesity on postoperative wound healing, and Penington and Morrison [12] reported that skin graft failure can be predicted by the waist-hip ratio, which is also related to being overweight. Wound healing can be compromised by obesity, diabetes, peripheral vascular disease, and smoking. We believe that some morbidities known as delayed wound healing factors can also affect the appearance of a scar. We have found that BMI, body weight, diabetes, and cardiac problems were significantly correlated with a negative aesthetic outcome. Other demographics or morbidities, including age, sex, history of smoking more than 10 packs per day, and defect size, were not correlated. The stepwise linear regression analysis showed that all of the correlated factors (BMI, body weight, diabetes, and cardiac problems) were significant independent factors associated with the aesthetic results. We also expected that peripheral vascular disease might have been a significant factor before we collected the data, but none of the patients had this condition. As a result, the aesthetic outcomes were much better than expected in patients who were not overweight or diabetic, or did not have cardiac problems. Several reports have been published about complications, such as unfavorable scarring on the donor site of a radial forearm flap; however, this is the first report on factors that can affect the aesthetics of scarring. Whether delayed wound healing is a compounding factor is controversial. However, of the 22 cases considered in the aesthetic evaluation, only one case had a wound healing problem; therefore, an analysis of the scar appearance is worthwhile.

A limitation of our study is that the evaluation of scarring and the data used for the statistical analysis were subjective because of the retrospective design of this study. The study was first designed on the basis of a comparison of other articles that mentioned the aesthetic results of the donor site of a radial forearm flap. All five of the other studies considered used the VAS score, so we also followed it for the sake of an easy comparison $[3,19$ 22]. Moreover, there are many studies in the literature that support the value of the VAS score and its reliability on the basis of a review of digital photographs for the scar appearance. Duncan et al. [14] explained that the VAS and scar ranking methods have been shown to meet the required standards of consistency, reliability, validity, and feasibility. In addition, they have been shown to have a high level of sensitivity in the clinical spectrum of scars thus assessed. Other articles also support the reliability of the VAS score. The VAS score based on pigmentation, vascularity, acceptability, observer comfort, and contour seems to be reliable enough for an evaluation of digital photographs [13-15]. The main disadvantages of using the radial forearm free flap include donor site complications, such as partial necrosis, sensory changes, and cosmetic problems with scarring and hyperpigmentation. However, the benefits of this flap easily outweigh these disadvantages, and its use should be considered for reconstructing head and neck defects. As described here, the possibility of donor site complications can be minimized by using meticulous procedures, and satisfactory aesthetic results can be achieved by using our results as a guideline for selecting candidates. If a candidate for a radial forearm flap is not overweight, does not have a high BMI, and is free of diabetes and cardiac problems, the aesthetic appearance can be excellent. Therefore, the anticipated donor site morbidity and aesthetic results should not be a reason to avoid using the radial forearm free flap as a coverage option for reconstruction in qualified cases.

\section{REFERENCES}

1. Yang G, Chen B, Gao W, et al. Forearm free skin flap transplantation. Natl Med J China 1981;61:139-41.

2. Evans GR, Schusterman MA, Kroll SS, et al. The radial forearm free flap for head and neck reconstruction: a review. Am J Surg 1994;168:446-50.

3. Richardson D, Fisher SE, Vaughan ED, et al. Radial forearm flap donor-site complications and morbidity: a prospective study. Plast Reconstr Surg 1997;99:109-15.

4. de Bree R, Hartley C, Smeele LE, et al. Evaluation of donor site function and morbidity of the fasciocutaneous radial forearm flap. Laryngoscope 2004;114:1973-6.

5. McGregor AD. The free radial forearm flap: the management of the secondary defect. Br J Plast Surg 1987;40:83-5.

6. Demirkan F, Chen HC, Wei FC, et al. The versatile anterolateral thigh flap: a musculocutaneous flap in disguise in head and neck reconstruction. Br J Plast Surg 2000;53:30-6.

7. Kuo YR, Jeng SF, Kuo MH, et al. Free anterolateral thigh flap for extremity reconstruction: clinical experience and functional assessment of donor site. Plast Reconstr Surg 2001; 107:1766-71.

8. Quillen CG. Latissimus dorsi myocutaneous flaps in head and neck reconstruction. Plast Reconstr Surg 1979;63:66470.

9. Hekner DD, Abbink JH, van Es RJ, et al. Donor-site mor- 
bidity of the radial forearm free flap versus the ulnar forearm free flap. Plast Reconstr Surg 2013;132:387-93.

10. Kim SW, Park SH, Koo SH, et al. Clinical applications of the radial forearm flap. J Korean Soc Plast Reconstr Surg 1997; 24:1090-9.

11. Wilson JA, Clark JJ. Obesity: impediment to postsurgical wound healing. Adv Skin Wound Care 2004;17:426-35.

12. Penington AJ, Morrison WA. Skin graft failure is predicted by waist-hip ratio: a marker for metabolic syndrome. ANZ J Surg 2007;77:118-20.

13. Fearmonti R, Bond J, Erdmann D, et al. A review of scar scales and scar measuring devices. Eplasty 2010;10:e43.

14. Duncan JA, Bond JS, Mason T, et al. Visual analogue scale scoring and ranking: a suitable and sensitive method for assessing scar quality? Plast Reconstr Surg 2006;118:909-18.

15. Micomonaco DC, Fung K, Mount G, et al. Development of a new visual analogue scale for the assessment of area scars. J Otolaryngol Head Neck Surg 2009;38:77-89.

16. Fenton OM, Roberts JO. Improving the donor site of the radial forearm flap. Br J Plast Surg 1985;38:504-5.

17. Wells MD, Kirn DS. A new method of skin-graft stabiliza- tion: the Reston technique. Ann Plast Surg 1995;34:554-6.

18. Saltz R, Bowles BJ. Reston: an alternate method of skin graft fixation. Plast Reconstr Surg 1997;99:601-2.

19. Lutz BS, Wei FC, Chang SC, et al. Donor site morbidity after suprafascial elevation of the radial forearm flap: a prospective study in 95 consecutive cases. Plast Reconstr Surg 1999;103:132-7.

20. Toschka H, Feifel H, Erli HJ, et al. Aesthetic and functional results of harvesting radial forearm flap, especially with regard to hand function. Int J Oral Maxillofac Surg 2001;30: 42-8.

21. Bootz F, Biesinger E. Reduction of complication rate at radial forearm flap donor sites. ORL J Otorhinolaryngol Relat Spec 1991;53:160-4.

22. Swanson E, Boyd JB, Manktelow RT. The radial forearm flap: reconstructive applications and donor-site defects in 35 consecutive patients. Plast Reconstr Surg 1990;85:258-66.

23. McCarthy JG. Plastic surgery. Philadelphia: Saunders; 1990.

24. Ragnell A. The secondary contracting tendency of free skin grafts; an experimental investigation on animals. Br J Plast Surg 1952;5:6-24. 